\title{
Risk of Hospitalization for Chronic Hemodialysis Patients
}

Katherine R. Jones

This study used primary data to analyze the incidence, causes and total days of hospitalization over a six-month period for a sample of chronic hemodialysis patients in one geographic area of the country. Patients were hospitalized most frequently for access-related and cardiovascular problems. Logistic regression analysis indicated that patients were more likely to be hospitalized if they had lower Karnofsky functional status scores, lower serum phosphate and protein levels, repeat access procedures, a negative hepatitis antigen, arthritis, psychiatric disorders, ischemic peripheral vascular disease or other cardiovascular conditions or were from larger households. Risk of hospitalization was not influenced by hemodialysis treatment characteristics. Recommendations for improved management of these high-cost patients were made, which could enhance quality of life and lower hospital-related costs.

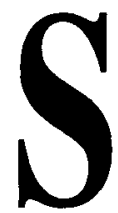

tudies have documented substantial case-mix differences among patients on hemodialysis (Eggers, 1982; Health Care Financing Administration [HCFA], 1987), but little work has been done so far on examining the direct effects of specific case-mix measures on the hospitalization experience of these patients (HCFA, 1987). This paper presents the results of an analysis of hospitalization in a sample of chronic hemodialysis patients, using primary data collected by medical record abstraction and review of treatment flow charts. Identification of specific risk factors for hospitalization could help in pinpointing those nursing and medical interventions that have the potential for reducing the incidence of hospitalization in this patient population. Reducing the level of morbidity might also help improve the quality of life of these chronically ill patients (Jones, 1991).

\section{Background}

Hemodialysis is a treatment that prolongs survival of the endstage renal disease patient but does not cure the underlying disease. Chronic hemodialysis allows the evolution of diseases which co-exist with chronic renal failure, such as diabetes mellitus and also creates a new clinical pathology (FunckBrentano, 1980).

Gral, Sokol and Rubini (1967) noted that hypertension, neuro- muscular disorders, skin and mucous membrane bleeding, pericarditis and metastatic calcifications are medical problems that may be relieved, but not cured, by the process of hemodialysis. In addition, anemia, peripheral neuropathy, skin pruritus and pigmentation, sterility and impotence, bone osteodystrophy and gout are problems that are usually unchanged by hemodialysis treatment. Medical problems caused by chronic hemodialysis include disequilibrium syndrome, dialysis dementia, electrolyte disorders, cardiovascular disorders such as uremic cardiomyopathy, pyogenic reactions, hepatitis, hematologic disorders, access problems and personality disorders, including psychosis (Funck-Brentano, 1980; Gral et al., 1967; Lindholm et al, 1985). It is obvious that an extensive range of variables would need to be included in an analysis of potential risk factors for hospitalization of the chronic hemodialysis patient.

Katherine R. Jones, RN, PhD, Rho, is Associate Professor, School of Nursing, University of Michigan. The author expresses her appreciation for the contributions made by the study advisory committee, the participating hemodialysis units and staff members, Catherine Repp, William Pfaff, Kerry Kilpatrick, Alice Privett, Dan Nissen, Harvey Evans and Paul Eggers. This research was supported by the Health Care Financing Administration, Contract \#14-C-98696/4-01. Correspondence to Division of Nursing and Health Care Systems Administration, School of Nursing, University of Michigan, $400 \mathrm{~N}$. Ingalls, Ann Arbor, MI 48109.

Accepted for publication November 7, 1991. 
Hospitalization is a recurring event in the chronic hemodialysis population (Delano, 1989; Pollak, Kant, Parnell \& Levin, 1986). Through their years of dialysis therapy, patients may require hospitalization for treatment of infection, cardiovascular instability, hypertension, myocardial infarction, gastrointestinal bleeding or a vast array of other conditions (Gutman \& Amara, 1978). The National Kidney Dialysis and Kidney Transplantation study (HCFA, 1987) reported that two-thirds of its samples were hospitalized at least once during the year. Patients being dialyzed in centers were hospitalized on average 2.4 times per year with an average of 6.6 days per episode and a mean total of 13.8 days per year. Blagg and Scribner (1980) noted that a large number of patients with severe complicating illnesses, some that even preclude living at home, have been admitted to chronic hemodialysis programs. Given the high cost of hospitalization, any progress made in identifying and reducing the risk associated with hospitalization in this population could result in substantial cost-savings, especially as the End-Stage Renal Disease (ESRD) population grows and becomes increasingly elderly and debilitated (Jones, 1991).

\section{Factors Associated with Hemodialysis Outcomes}

One major challenge in managing chronic hemodialysis patients is maintaining adequate nutrition. Poor nutritional status is reflected in lower albumin levels caused by increased catabolism and reduced dietary protein intake (Bianchi, Mariani, Guiseppina, \& Carmass, 1978). Significant losses of individual amino acids occur into the dialysate (Rubini \& Gordon, 1968), and the essential amini acid/non-essential amino acid ratio is reduced (Kopple, 1978), mimicking the findings of protein malnutrition (Faulks, 1988). Lowrie, Laird, Parker and Sargent (1981) found that higher blood-urea-nitrogen (BUN) levels and shorter dialysis times were associated with non-access-related hospitalization and concluded that patients who have restricted protein intake and are undernourished may also have a greater risk of medical complications and hospitalization.

Infection is a common cause of morbidity and mortality among chronic hemodialysis patients (Lewis, 1990). This is due in part to the need for vascular access and to abnormalities in the immune system of patients with ESRD. The most common sites of bacterial infections include vascular access, lungs and urinary tract (Keane, Shapiro \& Ray, 1977; Peresecenschi, Blum, Aviram \& Sperer, 1981).

Revision or replacement of the vascular access for hemodialysis treatments is occasionally required. The search for the "ideal" vascular access continues. External devices are subject to thrombosis, infection, dislodgement and skin erosion; internal fistulae complications include thrombosis, venous hypertension, ischemia, aneurysm, congestive heart failure and infection including sepsis (Parker \& Lancaster, 1984).

Hypotension is a frequent complication of the dialysis treatment, due to a decreased venous return to the heart by ultrafiltration-induced hypovolemia. This results in a decrease in filling pressures and cardiac output and left ventricular work index (Kinet et al, 1982). A decrease in plasma osmolality also contributes to hypotension (Rosa et al, 1981).

Depression and death have been associated with frequency of hospitalization (Numan, Barklind \& Lubin, 1981) and noncompliance may lead to hospitalization (Wolcott, Maida, Diamond \& Nissenson, 1986). Procci (1978) found that unemployed single males living away from their families are the most noncompliant group among hemodialysis patients and may therefore experience a greater number of hospitalizations.

The elderly experience a greater number of medical problems per patient, with lower blood pressure and serum phosphate levels, cardiac instability and more episodes of gastrointestinal bleeding, which may lead to more frequent hospitalizations (Chester et al., 1979). However, the elderly are also usually more compliant, which reduces risk of hospitalization (Czaczkes \& DeNour, 1978).

A primary diagnosis of diabetes mellitus and an increasing number of comorbid conditions increased hospital admissions and hospital days for patients in the National Kidney Dialysis and Kidney Transplantation Study (HCFA, 1987), while an increasing level of education reduced admissions and days. Functional status may also be an important predictor of hospitalization for this group of patients. Loss of function is related to the cumulative physical, physiologic and psychological effects of the disease process on the patient (Mor, Laliberte, Morris \& Weisman, 1984). Functional status has been used frequently as an outcome measure in comparisons of the different ESRD treatment modalities, but has not been used to predict risk of hospitalization (Evans et al, 1985; Hart \& Evans, 1987, HCFA, 1987).

The flat- or fixed-rate method of reimbursement for ESRD dialysis treatments are similar to diagnosis related groups (DRGs), in that they establish incentives for reducing the cost per dialysis treatment (Jones, 1991). Lack of patient-specific data, however, has made it difficult to evaluate the quality of care being delivered and to monitor patient responses to: cost-saving strategies of shorter dialysis times, lower skill mix, lower staffing ratios and reuse of dialyzers and tubes. It is possible that morbidity and mortality vary with treatment process and delivery system variations, but at this point little is known (Maxwell \& Sapolsky, 1987).

\section{Method}

The methods for the study have been described in detail elsewhere (Jones, 1991). It was conducted on a sample of 527 chronic hemodialysis patients who were dialyzing in seven different facilities, including proprietary, freestanding and hospital-based, not-for-profit units, in one geographic area of a Southeastern state from December 1985 through June 1986. In order to capture all available data on the maximum number of patients, all chronic hemodialysis patients dialyzing more than one month in each of the units were enrolled in the study.

Detailed patient case-mix information was collected on each patient by members of the hemodialysis nursing staff who were reimbursed for their efforts. Training sessions were conducted by a research associate who was also an experienced hemodialysis nurse clinician and educator. Forms were reviewed in detail and 
opportunity was provided for clarification of data entry processes and variable definitions. To further enhance the reliability of the data, a study coordinator in each unit looked after the distribution and collection of each data entry form, assigned patients to data collectors and reviewed the forms for completeness and accuracy. The research associate was available to the data collectors and study coordinators to answer questions and clarify information.

A variety of methods were used to identify variables that might be important in predicting hospitalization of the chronic hemodialysis patient. First, an extensive review was conducted of the nursing and medical literatures. Second, a national advisory committee consisting of physicians, nurses and casemix researchers provided both broad categories of variables and specific suggestions for data collection. Third, individual meetings were held with physicians and nurses working in hemodialysis units. And finally, the list of risk factors was reviewed for feasibility and comprehensiveness by the medical advisory committee of the regional ESRD network.

Sources of data were the medical record, hemodialysis treatment flow chart and the patient. The only subjective measurement was the Karnofsky Functional Status Scale (KPS), which required the nursing staff to judge the level of patient functioning (Hutchinson et al, 1979). Special training sessions with hypothetical cases were used to improve consistency in the assignment of KPS levels. In addition, the researchers advised the staff to discuss and assign the KPS scores as a group, which it did, in order to improve the consistency of judgments among nurses within individual units. No effort, however, was made to check the reliability of score assignments among the hemodialysis units in the study, so it is possible that some variation in score assignments may have occurred.

The Karnofsky Scale achieved face validity in this study, as members of the national advisory committee reviewed its content and agreed that it was the best tool available to measure relevant variations in functional status within the population of interest in this study. Construct validity was supported by finding that functional status scores were lower, on average, for patients in the older age groups (below $60 \mathrm{KPS}=63$; above 60 $\mathrm{KPS}=70 ; p=.0001$ ), in diabetics (KPS=61 versus $\mathrm{KPS}=69$ for nondiabetics, $\mathrm{p}=.0001$ ), in less educated patients (non-high school graduates KPS $=66$, high school graduates $\mathrm{KPS}=71$, $\mathrm{p}=.0052$ ), and in lower socioeconomic status (SES) patients (non-homeowners KPS $=64$, homeowners KPS $=69, \mathrm{p}=.0008$ ) (Jones, 1990).

Other categories of information collected were patient sociodemographic characteristics, primary renal diagnosis, specific cardiovascular, neurologic, gastrointestinal/metabolic and other comorbid conditions, compliance behaviors, specific risk factors and elements such as age and number of accesses and physiologic values. Information was also collected on the specific treatment procedure. Clinical, sociodemographic and treatment variables were collected at an initial time period and outcome measures were collected six months later. The list of variables, how they were measured and their expected relationship to the risk of hospitalization are shown in Table 1.

\section{Table 1: Description of Variables and Predicted Influence on Risk of Hospitalization}

\section{Sociodemographic information:}

Age (years): older $=$ increased risk

Race (white/black/other): unknown

Sex (male/female): male $=$ increased risk

Education (<h.s./h.s. grad/>h.s.): $<$ h.s. =increased risk

Household size (\#): unknown

Marital Status (married/single/widowed or divorced): married=reduced risk

Housing Status (homeowner/renter/other): homeowner less risk Medicaid (yes/no): yes=increased risk

Private health insurance (yes/no): yes=reduced risk

Functional status:

Karnofsky Performance Scale: higher levels=reduced risk

Comorbidities:

Cardiovascular (list of major diagnoses): presence $=$ increased risk

Gl/Metabolic (list of major diagnoses): presence $=$ increased risk Neurologic (list of major diagnoses): presence $=$ increased risk Other (list of major diagnoses): presence=increased risk

\section{Risk Elements:}

Months on hemodialysis: longer $=$ increased risk

History of CCPD, CAPD, Home (yes/no): yes=increased risk

History of transplant (yes/no): yes=increased risk

Diabetic history (months): longer=increased risk

Hepatitis antigen/non-A non-B hepatitis (yes/no): yes=increased $r$ isk

Residual Urine Function (yes/no): yes=reduced risk Cardiac arrhythmias (yes/no): yes=increased risk Accesses (\#): more $=$ increased risk

Primary Renal Disease:

Diabetes mellitus (yes/no): yes=increased risk

Hypertensive nephropathy (yes/no): yes=increased risk

Polycystic kidney disease (yes/no): yes=reduced risk

Glomerulnephritis (yes/no): unknown

Interstitial nephritis (yes/no): unknown

Collagen Disease (yes/no): increased risk

Other/Unknown (yes/no): increased risk

Compliance Behaviors:

No-shows (\#): more=increased risk

Early discontinue (\#): more=increased risk

Physiologic Values:

Potassium (meq) : higher=increased risk

Sodium (mg): lower=increased risk

Phosphate (mg): higher=increased risk

Calcium (mg): lower=increased risk

Albumin (mg): lower=increased risk

Protein $(\mathrm{mg})$ : lower=increased risk

Hematocrit $(\%)$ : lower=increased risk

BUN (mg): higher=increased risk

Pretreatment blood pressures $(\mathrm{mmH})$ : higher=increased risk

Posttreatment blood pressures $(\mathrm{mmHg})$ : lower=increased risk

Treatment Characteristics:

Hours per week: more=lower risk

Routine medications (\#): more $=$ increased risk

Type of access: external=increased risk

Artificial kidney (fiber/plate/coil): fiber=reduced risk

Dialysate delivery system (centralized/individual): individual=reduced risk

Dialyzer reuse: reuse=increased risk 
Table 2: Description of Chronic Hemodialysis Patients in the Study Sample.

\begin{tabular}{|c|c|c|}
\hline \multirow[t]{3}{*}{ Race: } & White & $30.9 \%$ \\
\hline & Black & $67.4 \%$ \\
\hline & Other & $1.7 \%$ \\
\hline \multirow[t]{2}{*}{ Sex: } & Male & $41.5 \%$ \\
\hline & Female & $58.5 \%$ \\
\hline \multirow[t]{4}{*}{ Marital: } & Married & $43.4 \%$ \\
\hline & Single & $13.1 \%$ \\
\hline & Divorced & $20.4 \%$ \\
\hline & Widowed & $23.0 \%$ \\
\hline \multirow[t]{4}{*}{ Education: } & Below High School & $59.2 \%$ \\
\hline & High School Graduate & $21.6 \%$ \\
\hline & Some College & $10.2 \%$ \\
\hline & College Graduate & $9.1 \%$ \\
\hline \multirow[t]{3}{*}{ Housing: } & Homeowner & $51.4 \%$ \\
\hline & Renter & $26.1 \%$ \\
\hline & Live with Others & $22.6 \%$ \\
\hline \multicolumn{2}{|c|}{ Medicaid: } & Yes $40 \%$ \\
\hline \multicolumn{2}{|c|}{ Private health insurance: } & es $35.5 \%$ \\
\hline \multicolumn{2}{|c|}{ Employed: } & es $12.9 \%$ \\
\hline \multicolumn{2}{|c|}{ Karnofsky < 70: } & $41.6 \%$ \\
\hline \multicolumn{2}{|c|}{ Age > 65: } & $31.8 \%$ \\
\hline
\end{tabular}

\section{Results}

Two-hundred forty (240) patients were from freestanding units and 287 were from hospital-based outpatient hemodialysis units. Descriptive results are shown in Table 2, and described in detail elsewhere (Jones, 1991). The majority of study participants were black, female and had less than a high school education. A large percentage were over the age of 65 and only 58 percent were judged able to carry on normal activity or to do active work.

\section{Frequency and Causes of Hospitalization}

Hospitalization is a frequent event in this population with 47.5 percent of the freestanding unit patients and 52.3 percent of the

Table 3: Description of Single Diagnosis Hospitalizations by Diagnostic Category, Total Days and Numbers of Episodes

\begin{tabular}{rrrr}
\hline Diagnostic Category & Number of Episodes & Number of Days & Mean Days \\
\hline Access problems & 113 & 1,063 & 9.4 \\
Cardiovascular & 65 & 554 & 8.5 \\
Gastrointestinal & 45 & 441 & 9.8 \\
Pulmonary & 30 & 252 & 8.4 \\
Other minor & 25 & 210 & 8.4 \\
Orthopedic & 22 & 231 & 10.5 \\
Neurologic & 21 & 167 & 7.9 \\
Other major & 19 & 182 & 9.6 \\
Electrolyte & 15 & 60 & 4.0 \\
Psychiatric & 8 & 111 & 13.9 \\
Metabolic & 7 & 99 & 14.1 \\
\hline Total & 370 & 3,370 & - \\
\hline
\end{tabular}

hospital-based unit patients being hospitalized at least once during the six-month follow-up period. The total group averaged 1.9 episodes per patient hospitalized. There were 500 hospital episodes and 3,370 days of hospitalization. The range of stays extended from one to 87 days, with an average hospital stay of 15.2 days.

More than one-half the episodes involved a single discharge diagnosis. As shown in Table 3, the greatest portion of single diagnosis hospital admissions was for access-related problems. Over 25 percent of the admissions were for access declotting or replacement, treatment of infection or rupture, or for placement of a catheter in preparation for peritoneal dialysis treatment.

The second most frequent reason for admission to the hospital was circulatory or cardiovascular-related problems, which is consistent with the high incidence of cardiovascular comorbid conditions in these patients. The hospitalizations involved episodes of congestive heart failure, hypertension, digitoxin toxicity, pericarditis and myocardial infarction. Gastrointestinal or metabolic disorders were the third most frequent cause of hospitalization, with diagnoses of gastrointestinal bleeding, pancreatitis and ulcers.

The remaining hospital episodes were distributed among neurologic disorders, pulmonary and orthopedic problems, electrolyte imbalances, other major diagnoses (sepsis, removal of kidney graft, cancer therapy) and other minor diagnoses (biopsies, fever, anemia and cataract repair).

\section{Bivariate Relationships}

The following variables were determined to have a statistically significant association with hospitalization, using student t-tests or Mann-Whitney U tests as appropriate and chi-square analysis. Patients experiencing hospitalization came from larger households and were less likely to be a homeowner or married. Hospitalized patients had lower functional ability and were found more frequently in the KPS categories below 70 . They required more accesses over time, were less likely to have a positive hepatitis antigen test result and had a higher total number of comorbid conditions. The specific comorbid conditions that achieved statistical significance were ischemic peripheral vascular disease, other cardiovascular conditions, psychiatric disorders, arthritis and systemic infection.

The hospitalized patients had lower serum protein levels and higher interdialytic weight gains. They also had received more blood transfusions.

\section{Multivariate Relationships}

Variables identified in the bivariate analysis as having a significant or nearly significant relationship with the occurrence of hospitalization were entered into multivariate models using logistic regression. The SAS (1990) logistic regression procedure was used to identify which variables had significant associations with the odds of being hospitalized (defined as a dichotomous dependent variable), holding other variables constant. Several models were estimated. In addition, the initial model specifications were later expanded to include all case-mix variables included in the list of risk factors for hospitalization, in order to determine whether variables not having a significant association 


\begin{tabular}{|c|c|c|c|c|c|}
\hline Variable & Beta & S.E. & $x^{2}$ & $\mathbf{P}$ & Odds Ratio \\
\hline Intercept & 1.089 & 1.993 & 0.30 & .5848 & - \\
\hline Arthritis: absent & -1.206 & 0.422 & 8.15 & .004 .3 & 3.34 \\
\hline$\#$ Accesses & 0.296 & 0.116 & 6.51 & .0117 & \\
\hline $\begin{array}{l}\text { Predialysis } \\
\text { Diastolic } \\
\text { B.P. }\end{array}$ & -0.107 & 0.039 & 7.68 & .0056 & $\begin{array}{l}1.35 \\
1.11\end{array}$ \\
\hline $\begin{array}{l}\text { Postdialysis } \\
\text { Diastolic } \\
\text { B.P. }\end{array}$ & 0.084 & 0.045 & 3.54 & .0601 & 1.09 \\
\hline $\begin{array}{l}\text { Model } \text { Chi-Square }=27.59 \\
\text { Correct: } 90.7 \%\end{array}$ & \multicolumn{3}{|c|}{$P=0.0$} & \multicolumn{2}{|c|}{$R=.302$} \\
\hline
\end{tabular}

with hospitalization in bivariate analyses might have a significant association when other variables are held constant in the models. Results did not change as a result of this additional analysis and those results are not presented here.

\section{Access-Related Hospitalization}

As seen in Table 4, the model for a single, access-related hospitalization predicted correctly 90.7 percent of the cases. Its specificity ( 99.6 percent), however, was superior to its sensitivity (3.6 percent). The model chi-square was 27.59 ( $p<.05$ ), indicating that the model did not fit the data well and more relevant variables remain to be detected and included. Four variables had a statistically significant relationship with access-related hospital episodes. Arthritis increased the odds, as did an increasing number of access procedures in the past and higher average postdialysis diastolic blood pressures. On the other hand, increasing average predialysis diastolic blood pressures reduced the odds of an access-related hospitalization.

The magnitude of influence exerted by these variables can be determined through calculation of the variable's odds ratio. The odds ratio indicates the relative risk of hospitalization associated with a given measure. For a dichotomous measure, the odds ratio represents the extent to which the chance of hospitalization is greater for a hemodialysis patient with the trait compared to a patient without the trait. For a continuous variable, the odds ratio represents the increased risk of hospitalization associated with each additional unit value. The odds ratios can be used to rankorder the model variables in terms of decreasing influence on risk of hospitalization. Looking at the odds ratios for the accessrelated risk variables, a patient with arthritis has a 3.34:1 greater chance of requiring an access-related hospitalization as compared to a patient without arthritis. Each addition access procedure in the past increased the odds of hospitalization for another access-related problem by 35 percent $(O . R .=1.35)$.

\section{Hospital Episodes}

As seen in Table 5, the logistic regression model for hospital admission predicted correctly 65 percent of the cases, with a specificity of 70.6 percent and a sensitivity of 58.9 percent. The model chi-square was again statistically significant, indicating a lack of fit and missing variables. Eleven variables had a significant association with the odds of being hospitalized. Variables
Table 5: Logistic Regression Results for Odds of Being Hospitalized, Using Specific Comorbid Conditions

\begin{tabular}{|c|c|c|c|c|c|}
\hline Variable & Beta & S.E. & $x^{2}$ & $\mathbf{P}$ & Odds Ratio \\
\hline Intercept & 6.169 & & & & \\
\hline Karnofsky & -0.020 & .006 & 11.34 & .0008 & 1.02 \\
\hline Arthritis: absent & -0.669 & .211 & 10.03 & .0015 & 1.95 \\
\hline Household size & 0.269 & .065 & 17.34 & .0000 & 1.31 \\
\hline Other Cardiovascular disease: absent & -1.157 & .463 & 6.23 & .0125 & 3,18 \\
\hline + Hep. Antigen: absent & 1.849 & .622 & 8.83 & .0030 & 6.35 \\
\hline Phosphate & -0.086 & .043 & 3.97 & .0464 & 1.09 \\
\hline Psychiatric illness: absent & -1.003 & .405 & 6.15 & .0131 & 2.73 \\
\hline Accesses & 0.164 & .077 & 4.48 & .0342 & 1.18 \\
\hline Protein & -0.302 & .156 & 3.77 & .0521 & 1.35 \\
\hline Interdialytic Wt & -0.101 & .048 & 4.48 & .0343 & 1.11 \\
\hline IPVD: absent & -0.634 & .303 & 4.38 & .0364 & 1.89 \\
\hline Model Chi-Square $=75.83$ & $P=0.0$ & & \multicolumn{2}{|c|}{$R=.297$} & \\
\hline Correct: $65.0 \%$ & \multicolumn{2}{|c|}{ Sensitivity: $58.9 \%$} & \multicolumn{3}{|c|}{ Specificity: $70.6 \%$} \\
\hline
\end{tabular}

that reduced the odds of being hospitalized were increasing functional status scores, higher serum phosphate levels, higher serum protein levels and greater interdialytic weight gain. Those factors associated with greater odds of being hospitalized were arthritis, larger household sizes, other cardiovascular conditions, negative hepatitis antigens, psychiatric disorders, greater number of vascular accesses over time, and ischemic peripheral vascular disease. The odds ratios indicated that negative hepatitis antigen had the largest influence on the outcome, being associated with six-fold increase in the odds of being hospitalized. Perhaps this reflects reluctance to hospitalize hepatitis patients. It may also be a statistical quirk caused by the very small number of hepatitis patients in the sample.

Having another cardiovascular condition (heart failure, cardiomyopathy, cardiomegaly, pacemaker) was associated with a three-fold increase in the odds of hospitalization, followed by a 2.7:1 increase in the odds for patients with psychiatric disorders, compared to not having these respective complications. Arthritis and ischemic peripheral vascular disease demonstrated a near two-fold increase in the odds of being hospitalized as compared to not having these comorbid conditions.

\section{Discussion}

Hospitalization is related, in part, to the treatment process itself, especially the requirement for vascular access. Number of vascular accesses was both a major cause of hospitalization in this study and also associated with hospitalization for non-access related problems. Nursing interventions and educational strategies directed at improving this risk factor include avoiding extremes of hot and cold, avoiding trauma to the access site, meticulous daily shunt care, constant covering/dressing over the shunt, appropriate needle insertion technique, rotation of sites, avoiding recirculation of blood by placing needles at least 1 and $1 / 2$ inches apart, observing for signs of infection and aneurysm formation and avoiding constriction of the extremity from tight clothing or carrying heavy dangling items such as a purse (Parker \& Lancaster, 1984). 
Increased attention to two factors may lead to fewer accessrelated hospital episodes: better blood pressure control and better management of arthritis patients. It appears important to avoid abnormally low diastolic blood pressures between dialysis treatments, while removing a sufficient amount of fluid and adequately lowering diastolic blood pressures during treatments. However, the low blood pressures may also be related to patient age. Chester et al. (1979) found in a small sample of patients that diastolic blood pressure declined with age, a decline that accelerated in the elderly. In fact, lower diastolic pressures, interdialytic weight gains and serum phosphate levels are all found more frequently in older patients (Chester et al., 1979), which may help explain the somewhat counter-intuitive results that occurred in this study (higher phosphate and weight gains associated with reduced risk) and why age itself was not a significant risk factor for hospitalization (Jones, 1991).

Arthritis patients may also require increased attention by clinicians. They may require special consideration in selection of access sites, type of access, monitoring during and between treatments, physical therapy and intensity of teaching and selfcare programs.

Cardiovascular problems and psychiatric disorders were found to be major reasons for hospitalization, which is consistent with other reported studies (Hutchinson, Thomas \& MacGibbon, 1982; Kutner \& Cardenas, 1981; Lindner, Charra, Sharrard \& Scribner, 1974; Lowrie, Lazarus, Hampers \& Merrill, 1974; Numan et al., 1981; Plough \& Salem, 1982). The results suggest that improved clinical management of anxiety, depression, heart block and ischemic peripheral vascular disease may reduce the morbidity associated with these conditions. Managing the anxiety and depression falls largely into the nurses' domain. Assisting patients to adapt to ESRD requires nurses to understand and use therapeutic communication skills. Therapeutic communication is characterized by goal-directed and purposeful exchanges, a focus on the present, encouragement and nurturing of expression of feelings, acknowledgement of individual self-worth and clarity of roles (Ulrich, 1980). The nurse provides careful and deliberate guidance. Psycho-social needs may include resolution of dependency/independency feelings, adjustment to role change and coping with crisis events such as access problems (Ulrich, 1989).

Chronic fatigue, common in ESRD patients, is related to both psychologic and physiologic factors such as anemia, altered metabolic state, anxiety, stress, depression, frustration and role conflict (Srivastava, 1986). Moreover, physical ability has been demonstrated to be a strong indicator of perceived quality of life (Stegman, Duncan, Pohren \& Sandstrom, 1985). In addition to therapeutic communication, more emphasis on rehabilitation and physical therapy, as recommended by Gutman, Stead \& Robinson (1981) and Evans, et al. (1985), as well as administration of irecombinant human erythropoietin (Hu-EPO), could lead to higher functional and emotional status levels and reduced hospitalizations.

Avoidance of protein depletion is also recommended, along with additional dietary counseling and nutritional supplements. In the National Cooperative Dialysis Study, patients with worse outcomes had low protein intake $(<.8 \mathrm{mg} / \mathrm{kg} /$ day $)$ and low energy stores (body fat) (Harter, 1983; Schoenfeld, Henry, Laird \& Rox, 1983). Patients with albumin levels below $3.5 \mathrm{gm} / \mathrm{L}$, transferrin below $200 \mathrm{mg} / \mathrm{dl}$, total lymphocyte count below 1500 cells $/ \mathrm{m}^{3}$, and a nonreactive skin antigen test should be placed on an aggressive program of nutritional support (Salmond, 1980), Albumin levels below $2.8 \mathrm{gm} / \mathrm{L}$ or total protein $<5.0 \mathrm{gm} / \mathrm{L}$ reflect severe visceral protein depletion. The nurse needs to assess the patient's energy reserves - if the patient has experienced involuntary weight loss of 10 percent or more of usual body weight in one year, or if the patient weighs less than 85 percent of ideal body weight, the patient is a candidate for aggressive nutritional support (Salmond, 1980).

Nurses can encourage increased oral intake either with enriched foods or supplements and intradialytic parenteral nutrition can be considered. Heidland and Kult (1975) demonstrated that intravenous amino acids and carbohydrates given during the last 90 minutes of dialysis can significantly increase albumin, total protein, transferrin and complement levels. Faulks (1981) notes that repletion occurs during a four-to six-month period, with regained appetite and physical strength, as well as a decrease in hospitalization rate. The addition of glucose to the dialysate decreases losses of amino acids (Kopple et al, 1973). Hemodialysis patients require a protein intake of at least 1.1 to $1.2 \mathrm{gm} / \mathrm{kg} / \mathrm{day}$ (Schoenfeld et al., 1983). If the patient is stressed or activity level increases, the protein intake should also increase. The anemiarelated pattern of poor appetite, lethargy and decreased mental and physical status can be reversed as the anemia is corrected by epoietin alfa. Then as appetite improves the need switches to monitoring of protein and potassium levels as appetite improves (Hensley, 1990).

None of the treatment variables examined in this study were found to have a significant association with risk of hospitalization. Reuse of dialyzers, centralized dialysate delivery systems and fewer hours of treatment per week did not increase a patient's risk of hospitalization, at least in the short-term.

The results of this study may not be generalizable to the rest of the chronic hemodialysis population. The sample of patients differed substantially in sociodemographic characteristics and other measures from those reported by HCFA. Of the national ESRD population, 23.2 percent are over the age of 65,56 percent are male and 68 percent are white. The national sample has been dialyzing on average a longer period of time than the study sample and are better educated. In addition, the study sample was drawn from one geographic area of one state. Additional studies need to be done on a more representative sample of patients, with data abstracted by trained individuals from outside the hemodialysis units and with appropriate reliability testing. Finally, more work needs to be done to identify all the important variables to consider in estimating the odds of being hospitalized. Model statistics indicate that relevant predictor variables that would improve the fit of the model to the data have yet to be determined.

\section{Conclusion}

Hospitalization is an outcome for the ESRD hemodialysis population that might be reduced in frequency through improved medical and nursing management of the patient. Specific inter- 
ventions that might lead to avoided hospitalizations include administration of $\mathrm{Hu}-\mathrm{EPO}$, dietary counseling and nutritional supplements to avoid protein depletion, avoidance of extremely low diastolic blood pressures between scheduled treatments, psychological counseling and psychiatric referrals and closer monitoring of depressed, anxious, heart block, arthritis and ischemic peripheral vascular disease (IPVD) patients. इएका

\section{References}

Bianchi, R., Mariani, G., Guiseppina, M. \& Carmoss, F. (1978). The metabolism of human serum albumin in renal failure on conservative and dialysis therapy. American Journal of Clinical Nutrition, 3, 1615-1626.

Blagg, C.R. \& Scribner, B.H. (1980). Long-term dialysis: Current problems and future prospects. American Journal of Medicine, 68, 633-635.

Chester, A.C., Rakowski, T.A., Argy, W.P., Giacalone, D. \& Schreiner, G.E. (1979). Hemodialysis in the eighth and ninth decades of life. Archives of Internal Medicine, 139, 1001-1005.

Czaczkes, J.W. \& DeNour, A.K. (1978). Chronic Hemodialysis as a Way of Life. New York: Brunner and Mazel.

Delano, B.G. (1989). Improvements in quality of life following treatment with $\mathrm{r}$-Hu-EPO in anemic hemodialysis patients. American Journal of Kidney Diseases, 14, 14-18.

Eggers, P.W. (1982). Analysis of indicators of case-mix differences between freestanding facility and hospital-based Medicare ESRD patients. Working Paper \#38. Baltimore, MD: Office of Research and Demonstrations, Health Care Financing Administration.

Evans, R.W.,Mannimen, D.L.,Garrison, L.P., Hart, L.G.,Blagg,C.L.,Gutman, R.A., Hull, A.R. \& Lowrie, E.G. (1985). The quality of life of patients with End-State Renal Disease. New England Journal of Medicine, 312, 553-559.

Faulks, R.J. (1988). Nutritional evaluation of patients on maintenance dialysis therapy. American Nephrology Nurses' Association Journal, 15, 13-17.

Funck-Brentano, J.L. (1980). New clinical pathology created by chronic dialysis. Advances in Nephrology, 9, 3-5.

Gral, T., Sokol, A., \& Rubini, M.E. (1967). Some medical problems of chronic hemodialysis. Israel Journal of Medical Science, 3, 14-27.

Gutman, R.A., \& Amara, A.H. (1978). Outcome of therapy for end-state uremia: An informal prediction of survival rate and degree of rehabilitation. PostGraduate Medicine, 64, 183-194.

Gutman, R.A., Stead, W.W. \& Robinson, R.R. (1981). Physical activity and employment status of patients on maintenance dialysis. The New England Journal of Medicine, 304, 309-313.

Hart, L.G., \& Evans, R.W. (1987). The functional status of ESRD patients as measured by the sickness impact profile. Journal of Chronic Diseases, 40, 117S-130S.

Harter, H.R. (1983). Review of significant findings from the National Cooperative Dialysis Study and recommendations. Kidney International, 23, 51075112.

Health Care Financing Administration (1987). Special Report: Findings from the National Kidney Dialysis and Kidney Transplantation Study. Baltimore, MD: Department of Health and Human Services.

Heidland, A. \& Kult, J. (1975). Long-term Effects of essential amino acid supplementation in patients on regular dialysis treatment. Clinical Nephrology, 3, 234-239.

Hensley, M.K. (1990). Case management of the anemic patient. American Nephrology Nurses' Association Journal, 17, 390-391.

Hutchinson, T.A., Boyd, N.F., Feinstein, A.R., Gonda, A., Hollomby, D. \& Rowat, B. (1979). Scientific problems in clinical scales, as demonstrated in the Karnofsky Index of Performance Status. Journal of Chronic Diseases, 32 , 661-666.

Hutchinson, T.A., Thomas, D.G. \& MacGibbon, B. (1982). Predicting survival in adults with End-State Renal Disease: An age equivalence index. Annals of Internal Medicine, 96, 417-423.

Jones, K.R. (1990). Functional Status in Chronic Hemodialysis Patients. Dialysis \& Transplantation, 19, 173-178.

Jones, K.R. (1991). Factors Associated with Hospitalization in a Sample of Chronic Hemodialysis Patients. Health Services Research, 26, 671-699.
Keane, W.F. Shapiro, F.L. \& Ray, L. (1977). Incidence and type of infections occurring in 445 chronic hemodialysis patients. Transactions of the American Society of Artinicial Organs, 23, 41-47.

Kinet, J.P., Soyeur, D., Balland, N., Saint-Remy, M., Collignon, P. \& Godon, J.P.(1982). Hemodynamic study of hypotension during hemodialysis. Kidney International, 21, 868-876.

Kopple, J.D. (1978). Abnormal amino-acid and protein metabolism in uremia. Kidney International, 14, 340-348.

Kopple, J.D., Swendseid, M.E., Shinaberger, J.H. \& Umezawa, C.Y. (1973). The free and bound amino acids removed by hemodialysis. Transactions of the American Society of Artificial Internal Organs, 19, 309-313.

Kutner, N.G. \& Cardenas, D.D. (1981). Rehabilitation status of chronic renal disease patients undergoing dialysis: variations by age category. Archives of Physical Medicine and Rehabilitation, 62, 626-630.

Lewis, S.L. (1990). Alteration of host defense mechanisms in chronic dialysis patients. American Nephrology Nurses' Association Journal, 17, 170-180.

Lindholm, T., Thysell, H., Yamamoto, Y. et al. (1985). Temperature and vascular stability in hemodialysis. Nephron, 39, 130-133.

Lindner, A., Charra, B., Sherrard, D.J. \& Scribner, B.H. (1974). Accelerated atherosclerosis in prolonged maintenance hemodialysis. The New England Journal of Medicine, 290, 697-701.

Lowrie, E.G., Lazarus, J.M., Hampers, C.L. \& Merrill,J.P. (1974). Cardiovascular disease in dialysis patients. The New England Journal of Medicine, 210, 737-738.

Lowrie, E.G., Laird, N.M., Parker, T.F. \& Sargent, J.A. (1981). Effect of the hemodialysis prescription on patient morbidity. The New England Journal of Medicine, 305, 1176-1181.

Maxwell, J. \& Sapolsky, H. (1987). The first DRG: Lessons from the End-Stage Renal Disease program for the Prospective Payment System. Inquiry, 24, 57 . 67.

Mor, V., Laliberte, L., Morris, J.N., \& Weimann, M. (1984). The Karnofsky Performance Status Scale: An examination of its reliability and validity in a research setting. Cancer, 53, 2002-2007.

Numan, I.M., Barklind, K.S. \& Lubin, B. (1981). Correlates of depression in chronic dialysis patients: Morbidity and Mortality. Research in Nursing \& Health, 4, 295-297.

Parker, S.R., \& Lancaster,L.E. (1984). Access to the circulation for hemodialysis. In L.E. Lancaster, (ed.) The Patient With End-Stage Renal Disease, 145-169. New York: John Wiley \& Sons.

Peresecenschi, G., Blum, M., Aviram, A. \& Sperer, Z.H. (1981). Impaired neutrophil response to bacterial infection in dialyzed patients. Archives of Internal Medicine, 141, 1301-1303.

Plough, A.L. \& Salem, S. (1982). Social and contextual factors in the analysis of mortality in End-Stage Renal Disease patients: Implications for health policy. American Journal of Public Health, 72, 1293-1295.

Pollak, V.E., Kant, K.S., Parnell, S.L. \& Levin, N.W. (1986). Repeated use of dialyzers in safe: Long-term observations on morbidity and mortality in patients with End-Stage Renal Disease. Nephron, 42, 217-233.

Procci, W.R. (1978). Dietary abuse in maintenance hemodialysis patients. Psychosomatics, 19, 16-24.

Rosa, A., Shideman, J., McHugh, R., Duncan, D. \& Kjellstrand, C.M. (1981). The importance of osmolality fall and ultrafiltration rate on hemodialysis side effects. Nephron, 27, 134-141.

Rubini, M.E., \& Gordon, S. (1968). Individual plasma-free amini acids in uremic: Effect on hemodialysis. Nephron, 5, 339-351.

Salmond, S.W. (1980). How to assess the nutritional status of acutely ill patients American Journal of Nursing, 80, 922-924.

SAS Institute, Inc. (1990). SAS Technical Report: P200. Version 6.04. Carey, N.C.

Schoenfeld, P.Y., Henry, R.R., Laird, N.M. \& Roxi, D.M. (1983). Assessment of nutritional status of the National Cooperative Dialysis Study. Kidney International, 23, 580-588.

Srivastana, R. (1986). Fatigue in the renal patient. American Nephrology Nurses' Association Journal, 12, 244-264.

Stegman, M., Duncan, C., Pohren, E. \& Sandstrom, S. (1985). Quality of life: A patient's perspective. American Nephrology Nurses' Association Journal, 12, 244-264.

Ulrich,B.T. (1989). Nephrology Nursing: Concepts \& Strategies. East Norwalk, Connecticut: Appleton \& Lange.

Wolcott, D.L., Maida, C.A., Diamond, R. \& Nissenson, A.R. (1986). Treatment compliance in End-Stage Renal Disease patients on dialysis. American Journal of Nephrology, $6,329-S 338$. 